\title{
Factors associated with preterm labor in Hormozgan province in 2013
}

\author{
Nasibeh Roozbeh ${ }^{1}$, Soheila Moradi ${ }^{2}$, Shirin Soltani², Fatemeh Zolfizadeh ${ }^{3}$, Mehdi Tadris Hasani ${ }^{3}$, Asiyeh \\ Pormehr Yabandeh ${ }^{4}$
}

${ }^{1}$ Ph.D. of Reproductive Health, Mother and Child Welfare Research Center, Hormozgan University of Medical Sciences, Bandar Abbas, Iran

${ }^{2}$ B.Sc. of Midwifery, Deputy of Health, Hormozgan University of Medical Sciences, Bandar Abbas, Iran

${ }^{3}$ B.Sc. of Family Health, Deputy of Health, Hormozgan University of Medical Sciences, Bandar Abbas, Iran

${ }^{4}$ M.Sc. of Midwifery, Instructor of Obstetrics Department, Mother and Child Welfare Research Center, Hormozgan University of Medical Sciences, Bandar Abbas, Iran

\section{Type of article: Original}

\begin{abstract}
Introduction: Preterm labor is the main cause of infants' death, and the main reason for undesirable consequences of pregnancy which still occur in some births, despite all of the interventions in this regard. The aim of this study was to evaluate every preterm birth and determine its maternal risk factors in Hormozgan province in 2013.

Methods: This case-control study was conducted in 2013 on the health and hospital records of 735 pregnant mothers with preterm labor (20-37 weeks) which were located in Bandar Abbas in southeast Iran. The sampling method would be multi-stage cluster sampling. The data collecting tool was a valid questionnaire by the Iran Ministry of Health and Medical Education, which included 5 components (demographic information, checking the current pregnancy of the mother, the maternal factors related to preterm labor in the previous pregnancy, evaluating the fetal factors associated with preterm labor in the current pregnancy and other additional information related to the factors associated with prematurity). Data were analyzed using descriptive statistics, regression and Chi-square tests

Results: The prevalence of preterm labor in Hormozgan province in 2013 was $5.5 \%$. In our study and among the effective factors on preterm labor, revealed genital-urinary tract infections during pregnancy were $(35.8 \%)$, PROM (30.3\%), history of abortion (19.9\%) and unplanned pregnancy $(18.1 \%)$ in between pregnant women with preterm labor. Our study showed that most mothers had delivered in 33 to 37 weeks among the mothers who had preterm labor in $2013(75 \%)$ and the statistic of very preterm labor (less than 32 weeks) in Hormozgan was 5\%.

Conclusion: Identification of the prevalence and associated factors with preterm labor can reduce the mortality rate and infants' complications of it in addition to help find a way to prevent this problem.

Keywords: Preterm labor, Mothers, Infants
\end{abstract}

\section{Introduction}

Preterm labor is defined as the parturition before completion of 37 weeks of pregnancy (less than 259 days) and is considered as the reason for two-thirds of deaths in the first year of life. $5-9 \%$ of all births in the developing countries and an average of $12 \%$ of deliveries in the US are preterm labor (1). Preterm labor is a great risk in the way of women's health and an important factor in the incidence of infants' mortality all over the world (1). Its complications can be intraventricular hemorrhage, cerebral palsy, neurological complications or high costs, to name a few. As well as these, the complications due to other undesirable consequences of preterm labor, such as the incidence risk of infant sudden death, malnutrition, child abuse and the lack of adequate relationship between mother and child in premature infants have been more observed (2). Preterm labor is the main reason of infants'

\section{Corresponding author:}

Asiyeh Pormehr Yabandeh, Mother and Child Welfare Research Center, Hormozgan University of Medical Sciences, Bandar Abbas, Iran. Tel: +98.7633667904, Fax:+98.7633670724, Email: Asipormehr@yahoo.com

Received: November 01, 2015, Accepted: April 09, 2016, Published: September 2016

iThenticate screening: April 09, 2016, English editing: July 24, 2016, Quality control: August 04, 2016

(C) 2016 The Authors. This is an open access article under the terms of the Creative Commons Attribution-NonCommercialNoDerivs License, which permits use and distribution in any medium, provided the original work is properly cited, the use is non-commercial and no modifications or adaptations are made. 
morbidity and mortality and the main cause of undesirable consequences of pregnancy that despite of all the carried out interventions in this regard, still occurs in some births (3). The complications of preterm labor are common and $75 \%$ of mortality and complications of infant period is due to preterm labor (birth before 37 weeks) $(3,4)$. The prevalence of preterm labor in different regions is reported as $5-11 \%$ and in diseases during pregnancy especially diabetes reaches to $24 \%$, too (4). The recurrence likelihood of this problem in subsequent pregnancies is approximately $6-8 \%(5)$. There are several risk factors for preterm labor, including demographic factors such as age of mother (younger than 17 and older than 35 years are at higher risk for preterm labor), maternal race (patients with African origin face more than Caucasians with preterm labor), low socioeconomic level and its resulting malnutrition as well as inadequate care during pregnancy $(3,4)$ and also behavioral factors in mother such as smoking are related to preterm labor $(6,7)$. In a study which was conducted in the US, $10-20 \%$ of preterm labors had been related to the mother being a smoker (6). In cases of maternal weight gain during pregnancy, it has been determined that preterm labor is more common in women with a body mass index of less than $19.8(7,8)$. Regarding the history of premature labor, one and two previous preterm labors are associated with 3 and 6 -folds of preterm labors, respectively (7). Regarding the history of previous abortions and multiple pregnancies, the risk of preterm labor is also higher (8). Vaginal bleeding in the first or second trimester, because of placenta previa or abruption and also development of pre-eclampsia and diabetes, is associated with a higher frequency of preterm labor (9). The relative risk of preterm labor associated with bleeding is reported to be around $7.4 \%$ in the previous studies (8). Infections are the factors which can have a role in preterm labor. In cases of chorioamnionitis in the current pregnancy of a mother, the probability of preterm labor has been reported as $25 \%$ (7). Urinary tract infections or vaginitis especially bacterial vaginosis, are associated with an increased risk of $40 \%$ in preterm labor (3). PROM, defined as the Premature Rupture of amniotic Membranes, and occurs one hour before the start of uterine contractions, is associated with preterm labor, and vaginal colonization by infections during pregnancy, lead to an increased risk of PROM (10). Preterm labor is the most important cause of admission of mothers during pregnancy to hospitals, and can create economic problems for the family and society in general. (11). In spite of all efforts made in its prevention, risk of development is still increasing (4). According to the mutual relationship between prematurity and infant mortality, various studies have been considered regarding the prevalence of prematurity and its relationship with some special factors and shown its high prevalence (10-13). The greatest cause of death in children less than 5 years old in Hormozgan province is prematurity and since the related factors with preterm labor have not been studied in Hormozgan province in recent years, the raised data from the present study can provide information for interventions and health planning to improve children's health for directors and health experts. The purpose of this study was to determine the associated factors with preterm labor in Hormozgan province in 2013.

\section{Material and Methods}

This study is a retrospective cross-sectional one from 2013 in Hormozgan province. In order to determine the sample size, by considering $\delta=20, C I=95 \%, Z=2$, and $d=0.1 \partial$, the sample size was calculated to be 400 people; so, 400 people from rural communities and 400 people from urban communities were selected. Considering a $10 \%$ exclusion of samples, the total sample size was estimated as 800 people. The sampling method would be multi-stage cluster sampling (health houses in rural areas and urban health-care centers are considered as clusters). The number of samples in each cluster will be 10 people. In proportion to the number of health houses and health-care centers in each city, numbers of clusters are selected by a simple random sampling method in each cluster; samples would be selected by systematic random sampling based on the serial numbers for preterm labor, which are recorded in prenatal care offices. The research population is included in all files of rural and urban pregnant women in Hormozgan province in which the termination of pregnancy in 20-weeks to 37-weeks of pregnancy are recorded. The criterion of being a preterm fetus was the first day of the mother's last menstruation or the first ultrasound from less than 12 weeks of pregnancy, recorded in the files. The cases with partially filled information were excluded from the study. The data collecting toll was a valid questionnaire by Iran's ministry of health and medical education (the questionnaire was for evaluating the effective factors on prenatal death) consisting 5 categories (demographic information, checking the current pregnancy of the mother, the maternal factors related to preterm labor in previous pregnancy, evaluating the fetal factors associated with preterm labor in the current pregnancy and other additional information related to the factors associated with prematurity) which were completed by the responsible expert of child healthcare in the cities of Hormozgan province. In order to determine the validity of the questionnaire, the comments of 10 faculty members (Pediatricians, Obstetricians and Masters of Midwifery) were used. The reliability of the tool was determined by Cronbach's alpha coefficient. So that after filling 20 questionnaires Cronbach's alpha coefficient was calculated as 0.71 . In order to standardize questionnaire completion, the essential education regarding the questionnaire completion and extraction of information from the file was presented. After completion and collection of data, the questionnaires were reviewed and subsequently, data were entered into the computer and 
http://www.ephysician.ir

analyzed using SPSS software. To describe data, descriptive statistics, tables and graphs were used and to analyze data, regression and Chi-square statistical tests were used. The significance level was considered less than 0.05 .

\section{Results}

Finally, the health and hospital files of 735 pregnant mothers with preterm labor were evaluated. The prevalence of preterm labor in Hormozgan Province was 55\% in 2013. The average age of mothers with preterm labor was 31 years. Most of them were housewives, who lived in rural areas and had diplomas. Most infants were male (51\%). Most mothers delivered in hospital and in the normal way (51.6\%). The average body mass index of most mothers was in the normal range. Among the effective factors on preterm labor, genital-urinary tract infections during pregnancy (based on the physical exam by a midwife or obstetrician or the test report in the file), PROM, history of abortion and unplanned pregnancy were the most effective factors (Table 1). The average weight of fetuses had been $890 \mathrm{~g}$ which increased with progressive gestational age. Most births were first and second rank (73.1\%). The evaluated cases were divided into three groups of 20-28 weeks, 29-32 weeks and 33-37 weeks. Most mothers delivered in 33 to 37 weeks ( $75 \%$ ) and the lowest percentage of preterm labor was related to 20-28 weeks (8\%). The history of abortion among three groups of preterm labor had a significant difference and the history of abortion in the group 20-28 weeks was more than other groups. Multiple pregnancies in three groups had a significant difference. The women who delivered in weeks 29-32, had a greater history of twins. Different groups of preterm labor did not have a significant statistical difference in terms of pregnancy interval with each other and most of them had observed the 3-years interval.

Table 1. The effective factors on preterm labor

\begin{tabular}{|l|l|l|l|}
\hline Factors & $\mathrm{n}$ & $\%$ & $\mathrm{p}$-value \\
\hline Smoking & 22 & 3 & 0.618 \\
\hline Having underlying disease & 100 & 13 & 0.786 \\
\hline Infections during pregnancy & 263 & 35.8 & 0.125 \\
\hline Receiving incomplete pregnancy care & 80 & 10.9 & 0.677 \\
\hline Having a history of abortion & 146 & 19.9 & 0.008 \\
\hline Having the history of prematurity & 85 & 11.6 & 0.818 \\
\hline PROM & 223 & 30.3 & 0.482 \\
\hline Unplanned pregnancy & 133 & 18.1 & 0.208 \\
\hline Risky behavior & 15 & 2 & 0.916 \\
\hline Having a history of multiple pregnancies & 75 & 10.2 & 0.042 \\
\hline Oral diseases & 92 & 12.5 & 0.217 \\
\hline Use of infertility drugs & 38 & 5.2 & 0.182 \\
\hline
\end{tabular}

\section{Discussion}

Our study showed that during the year 2013, the prevalence of preterm labor in Hormozgan province was 5.5\%. The prevalence of preterm labor is much lower than the reported rate for Africa $(12.6 \%)$ and Asia $(9.8 \%)(14,15)$. The low rate of preterm birth in the present study can be due to the success of different plans introduced by the Ministry of Health in Iran for improving the quality of presented health services to the pregnant women, including care before pregnancy and health care during pregnancy in the last decade, but the studies which were conducted in Iran are consistent with our study. In the studies of Bayat Mokhtari et al. (2007-2008) and Ali Jahan et al. (2013) which were conducted in Qazvin and Ardebil, the prevalence of preterm labor was 6\% and 5\%, respectively $(16,17)$ and these studies were consistent with our study. But, the prevalence of preterm labor in Qazvin province is higher than Hormozgan province and this difference can be due to the higher statistics of live birth numbers and the higher ratio of preterm labor in Qazvin province. On the other hand, mothers' access to the units, and the experts equipped for the management of preterm labor, can be effective. In a study by Mirzaei et al. in 2007 in Kerman, the incidence of preterm labor was reported as $39 \%$ which was more than all of the evaluated studies (18). It seems that the sample size in Mirzaei's study was lower than our study and this difference in the numbers of preterm labors in Hormozgan and Kerman provinces can explain the higher prevalence of preterm labor in Kerman.

Our study showed that among the mothers with preterm labor in 2013, most of them were delivered at 33 to 37 weeks $(75 \%)$ and the statistics of every preterm labor (under 32 weeks) in Hormozgan was $5 \%$. In a study conducted by Mansour Ghanei et al., 70\% of preterm labors occur by delay (age 34-37) which is consistent with our study (19). In a Beigi et al. study in 2009 which was performed on 4393 childbirths in Arash Hospital of Tehran, the prevalence 
of every preterm labor was reported as $1.34 \%$ (20) which is different from our study. Due to the higher preterm labor statistics and number of births in Hormozgan province, this difference is justifiable. The much higher preterm labor statistics in the province may be due to lack of awareness of mothers regarding the risk factors during pregnancy, and late visiting of health centers. On the other hand, the fragmented population of Hormozgan province as well as the distance between mothers' homes with specialized hospitals led to lack of mother access to specialized services and in-time visit of hospitals to control and stop preterm labor. In our study, genital-urinary tract infections during pregnancy (35.8\%), PROM (30.3\%), history of abortion (19.9\%) and unplanned pregnancy (18.1\%) are the most effective factors on preterm labor among the other factors. In the Molina et al. study in 2008, asymptomatic bacteriuria is one of the most common causes of preterm labors that is consistent with our study (21). In Schieve et al. study, they considered urinary tract infection as a risk factor for the birth of premature infants (22). Urinary tract infections are the most common infection during pregnancy and infection may increase the release of inflammatory chemokines and cytokines such as interleukin and tumor necrosis factor (23). Microbial endotoxin and preinflammatory cytokines stimulate production of prostaglandins (other inflammatory mediators) and degrading enzymes which eventually lead to stimulate uterine contractions, premature rupture of membranes and preterm birth (24). In a study by Khalajinia et al. in 2006 on the related factors with preterm labor, PROM and unplanned pregnancy were related to preterm labor which is consistent with our study and confirms it (25). The second common cause of preterm labor after spontaneous contractions of the uterus is PROM and it occurs in $40 \%$ of preterm labors. Long-term smoking, the previous history of preterm labor, bleeding (at any time during pregnancy) and urinary tract infection, increase the risk of PROM (26). Women with unplanned pregnancies are twice as much at risk of preterm labor than women with planned pregnancies. Given that women with unplanned pregnancies avoid receiving pregnancy care and do not have appropriate nutritional behavior they are at risk of preterm labor (27). Our study showed that the history of abortion is one of the effective factors of preterm labor. In a Swengel et al. study in 2009 , they showed that there is a relationship between abortion and preterm labor (28). The study of Bayat Mokhtari et al., also showed the relationship between abortion and preterm labor (16). Given that it is possible that many effective factors on abortion including uterine abnormalities, chronic diseases such as diabetes, history of drinking alcohol can also be related to preterm labor, higher risk of preterm birth in people who have a history of abortion can be justified.

\section{Strengths and limitations of the study}

One of the strengths of our study is the actual subject of study. Given that no study had been conducted in Hormozgan province to identify the prevalence and associated factors with preterm labor, the identification of the prevalence and associated factors with preterm labor can reduce the mortality rate and infant complications of it; in addition to help finding a way to prevent this problem. Our study was a cross-sectional one and data were extracted from the records of mothers. Limitations to access the journals and articles was the other restriction of this study.

\section{Conclusions}

The study showed that the prevalence of preterm labor in Hormozgan province was $5.5 \%$ and the history of genitalurinary tract infections during pregnancy was associated to preterm labor more than other factors. Due to the high statistics of child mortality in Hormozgan province because of prematurity, understanding the prevalence and the associated factors with preterm labor in addition to helping to find a way to prevent this problem, can reduce the mortality rate and infant complications of it. On the other hand, identification of mothers with the symptoms of genital-urinary tract infections during pregnancy, on-time treatment and essential education in the field of prevention of preterm labor can be effective.

\section{Acknowledgments:}

This article is part of an Approved Project (project No. 9280) in Hormozgan University of Medical Sciences, Bandar Abbas, Iran. The authors express their gratitude to the colleagues at Hormozgan University of Medical Sciences for their assistance in this study. And all of those who helped conduct this study.

\section{Conflict of Interest:}

There is no conflict of interest to be declared.

Authors' contributions:

All authors contributed to this project and article equally. All authors read and approved the final manuscript. 


\section{References:}

1) Ancel PY. Preterm labor: pathophysiology, risk factors and outcomes. J Gynecol Obstet Biol Reprod (Paris). 2002; 31(7): 10-21. PMID: 12454622.

2) Negahban T, Rezaiean M, Jamali M, Ansari A. The Relation between Primary Dysmenorrhea and Spontaneous Preterm Labor in Primiparous Women in Rafsanjan. Iranian Journal of Obstetrics, Gynecology and Infertility. 2012; 14(7): 37-43.

3) Zaini BT. Risk Factor for Premature Delivery among women in Hospital Tuanku Jaafar, Seremban. Gynecol Obstet Mex. 2006; 80(10): 610-12.

4) Hillier SH. Common vaginal condition Increase Risk of preterm Delivery and low Birth Weight. NICHD. 1995; 301: 496-5133.

5) Jakobsson M, Gissler M, Paavonen J, Tapper AM. The incidence of preterm deliveries decreases in Finland. BJOG. 2008; 115 (1): 38-48. doi: 10.1111/j.1471-0528.2007.01565. PMID: 18053102.

6) Martin R, Fanaroff A, Walsh M, Lindsay M. Pregnancy complicated by diabetes mellitus, 8 ed. Philadelphia: Mosby publication. 2006; 322-39.

7) Viswanathan M, Siega-Riz AM, Moos MK, Deierlein A, Mumford S, Knaack J, et al. Outcomes of maternal weight gain. Evid Rep Technol Assess. 2008; 16(8): 1-223. PMID: 18620471, PMCID: PMC4781425.

8) Borkowski $\mathrm{W}$, Mielniczuk $\mathrm{H}$. The influence of social and health factors including pregnancy weight gain rate and pre-pregnancy body mass on low birth weight of the infant. Ginekol Pol. 2008; 79: 415-21. PMID: 18652129.

9) Jesse E, Seaver W, Wallace D. Maternal psychosocial risks predict preterm birth in a group of women from Appalachia. Midwifery. 2003; 19(3): 191-202. PMID: 12946335.

10) Krupa FG, Faltin D, Ceatti JG, Surita FGC, Souza JP. Predictors of preterm birth. Int J Gyn Obs. 2006; 9(4): 5-11. PMID: 12946335.

11) Namakin K, Sharifzade G. Evaluation of infant mortality causes and its related factors in Birjand. Journal of Isfahan Medical School. 2009; 57(95): 175-82.

12) Falahi M, Jodaki N. Causes of infant mortality Shohada hospitals in the years 2004 to 2007. Pajouhesh. 2009; 14(1): 43-6.

13) Al Hosani HA, Brebner J, Bener AB, Norman JN. Study of mortality risk factors for children under age 5 in Abu Dhabi. East Mediterr Health J. 2003; 9(3): 333-43. PMID: 15751926.

14) Goldenberg RL, Culhane JF, Iams JD, Romero R. Epidemiology and causes of preterm birth. The Lancet. 2008; 371(9606): 75-84. doi: 10.1016/S0140-6736(08)60074-4. PMID: 18177778.

15) Chiabi A, Mah EM, Mvondo N, Nguefack S, Mbuagbaw L, Kamga KK, et al. Risk Factors for Premature Births: A Cross-Sectional Analysis of Hospital Records in a Cameroonian Health Facility. Afr J Reprod Health. 2013; 17(4): 77-83. PMID: 24558784.

16) Bayat Mokhtari, Tabrizian N. Maternal risk factors in causing of preterm delivery. Journal of Medical Sciences, Mashhad Islamic Azad University. 2010; 3(19): 140-5.

17) Alijahan R, Hazrati S, Mirzarahimi M, Pourfarzi F, Ahmadi Hadi P. Prevalence and risk factors associated with preterm birth in the city of Ardabil, Iran. Iran J Reprod Med. 2014; 1(12): 47-56. PMCID: PMC4009588.

18) Mirzaie F, Mohammah-Alizadeh S. Contributing factors of preterm delivery in parturient in a University Hospital in Iran. Saudi medical journal. 2007; 28(3):400-4. PMID: 17334468

19) Mansourghanaei M. Seasonal pattern of preterm birth. J Guilan Univ Med Sci 2011; 20(79):77-83.

20) Beigi A, Taheri N, Norouzi $\mathrm{H}$. The prevalence of very preterm deliveries, risk factors, and neonatal complications in Arash women hospital: a brief report. Tehran Univ Med J. 2013; 71(3): 194-8.

21) Molina JP, Meza JG, Reyes JC, Barón GP, Hernández JF. Preterm delivery and asymptomatic bacteriuria. Ginecol Obstet Mex. 2008; 76(8): 454-60. PMID: 18798448.

22) Schieve LA, Handler A, Hershow R, Persky V, Davis F. Urinary tract infection during pregnancy: its association with maternal morbidity and perinatal outcome. Am J Public Health. 1994; 84(3): 405-10. PMCID: PMC1614832.

23) Dimetry SR, El-Tokhy HM, Abdo NM, Ebrahim MA, Eissa M. Urinary Tract Infection and Adverse Outcome of Pregnancy. J Egypt Public Health Assoc. 2007; 82(3): 210-18. PMID: 18410708.

24) Guaschino S, De Seta F, Piccoli M, Maso G, Alberico S. Aetiology of preterm labour: bacterial vaginosis. Bjog. 2006; 113: 46-51. PMID: 17206964.

25) Khalaji nia, Sadeghi. The incidence of maternal risk factors associated with preterm delivery in Qom, 2006. Qom University of Medical Sciences. 2010; 5(1): 30-6. 
26) Mancuso RA, Schetter CD, Rini CM, Roesch SC, Hobel CJ. Maternal prenatal anxiety and corticotropinreleasing hormon associated with timing of delivery. Psychosom Med. 2004; 66(5): 762-9. PMID: 15385704.

27) Orr ST, Miller CA, James SA, Babones S. Unintended pregnancy and preterm birth. Paediatr Perinat Epidemiol. 2000; 14(4): 309-13. PMID: 11101017.

28) Swingle HM, Colaizy TT, Zimmerman MB, Morriss FH, In MD. Abortion and the risk of subsequent preterm birth. J Reprod Med. 2009; 54(2):95-108. PMID: 19301572 